\title{
BANCO DE ENSAYO PARA MOTORES DE VEHÍCULOS ELÉCTRICOS
}

\author{
Jose Luis Torres-Moreno, Galo Heredia, Antonio Gimenez-Fernandez \\ Departamento de Ingeniería, CIESOL-ceiA3, Universidad de Almería. \\ jltmoreno@ual.es, galojheredia@gmail.com, agimfer@ual.es \\ Antonio Visioli
}

Department of Mechanical and Industrial Engineering, University of Brescia. antonio.visioli@unibs.it

\section{Resumen}

En este trabajo se presenta un novedoso banco de ensayos para motores de vehículos eléctricos diseñado para reproducir las condiciones de trabajo de un vehículo en términos de par y velocidad angular teniendo en cuenta su inercia equivalente. Este banco permite, por un lado, elaborar modelos tanto del motor como de las baterías, así como diseñar y probar controladores y por el otro, la integración un vehículo eléctrico en una microrred eléctrica experimental de modo que se puedan realizar ensayos y evaluar estrategias de control energético sin necesidad de disponer del vehículo real. A raíz de los resultados obtenidos, se puede concluir que el diseño propuesto cumple con los requerimientos preliminares.

Palabras clave: Banco de ensayos, vehículo eléctrico, control de motores.

\section{INTRODUCCIÓN}

Los vehículos eléctricos ligeros están desempeñando un papel importante en aspectos relacionados con movilidad urbana y gestión eficiente de la energía. Por un lado, este tipo de vehículos son mucho más eficientes desde un punto de vista energético y ambiental que los vehículos convencionales. Por otro lado, al estar dotados de un conjunto de baterías con una alta capacidad de almacenamiento de energía, estos vehículos pueden integrarse en una estrategia de control energético en microrredes mediante los denominados sistemas vehículo a red (V2G) y red a vehículo (G2V). Numerosos esfuerzos en investigación sobre estos sistemas se están llevando a cabo durante los últimos años $[7,8,2]$.

Para llevar a cabo ensayos experimentales sobre estrategias de control V2G es necesario disponer de un vehículo eléctrico, de modo que se puedan dimensionar tanto el consumo eléctrico para distintos tipos de trayectos como el comportamiento de las baterías tanto en la carga y descarga como en la capacidad para devolver energía a la microrred [11]. Sin embargo, el tiempo de desar- rollo de este tipo de experimentos, la puesta a punto del vehículo y la necesidad de ser llevados a cabo mediante conducción humana los convierte en un proceso costoso. Para simplificar esta labor, se puede recurrir a la utilización de un banco de ensayos y la aplicación de técnicas hardwarein-the-loop $[9,6,5]$. En algunos trabajos encontrados en la literatura no se tienen en cuenta la inercia del vehículo, a pesar de que, como se verá en la sección 2, esta juega un papel fundamental si se pretenden emular las condiciones de funcionamiento del vehículo real [4]. Existe una propuesta de modelado en la que se utiliza el concepto de masa equivalente, que tiene en cuenta además la inercia de los elementos de rotación, aunque no se llevaron a cabo estudios experimentales. Por otro lado, la posibilidad de contar con un banco de ensayos que reproduzca fielmente el comportamiento del motor de la misma forma que si éste opera en el vehículo real, permite el diseño y pruebas de diferentes tipos de controladores desde una fase temprana de desarrollo de vehículos, contemplando aspectos como el control de velocidad o la frenada regenerativa [10].

Teniendo en cuenta todo lo anterior, en este trabajo se propone el diseño de un banco experimental para el motor del vehículo eléctrico mostrado en la Figura 1 atendiendo a los siguientes requerimientos: (i) contar con un volante de inercia

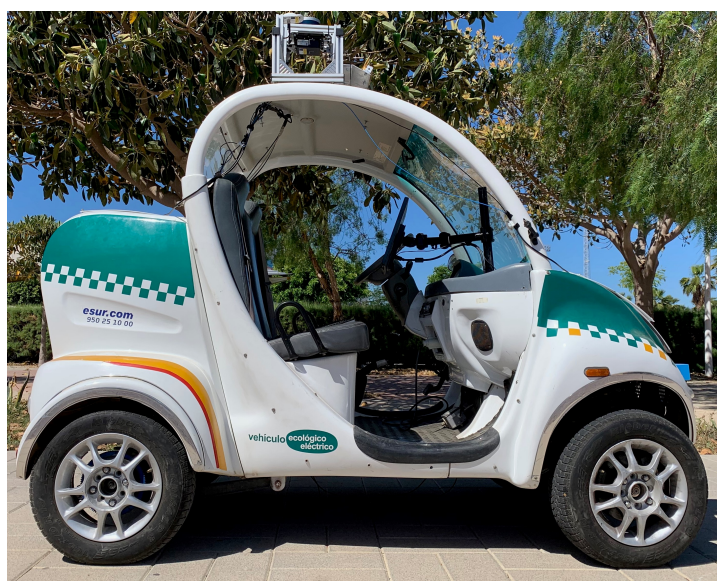

Figura 1: Vehículo eléctrico experimental eCARM 
equivalente a la del vehículo original en su desplazamiento longitudinal (ii) permitir que el motor trabaje en un régimen de par y velocidad similares a los del vehículo real y (iii) contar con un sistema de baterías que permita, por un lado, alimentar al motor a través de un amplificador y por otro permitir el flujo bidireccional de potencia en una microrred. Para ello, el resto de este artículo se estructura como sigue: en la sección 2 se presenta el modelado del vehículo eléctrico objeto de estudio con objeto de dimensionar justificadamente el volante de inercia que debe incorporar el banco de ensayos. En la sección 3 se presenta el diseño del mismo, teniendo en cuenta todos los requerimientos. En la sección 4 se presenta el banco construido y los resultados obtenidos en ensayos preliminares y finalmente en la sección 5 se presentan las conclusiones del trabajo desarrollado.

\section{MODELADO}

En esta sección se pretende dimensionar el volante de inercia que deberá incorporar el banco de ensayos con objeto de replicar las condiciones de funcionamiento en el vehículo real. Para ello, en primer lugar, es necesario conocer las fuerzas que éste debe vencer para llevar a cabo una trayectoria determinada. En segundo lugar, en base a la identificación de las fuerzas que afectan al vehículo, se llevarán a cabo los cálculos pertinentes para la definición de la inercia que debe incorporar el banco de ensayos.

\subsection{DINÁMICA VEHICULAR LONGITUDINAL}

En el estudio de la dinámica longitudinal de un vehículo se considera principalmente la aceleración de éste y su frenado, sin tener en cuenta el deslizamiento de los neumáticos, esto es, en condiciones de rodadura pura. Además de esta simplificación,

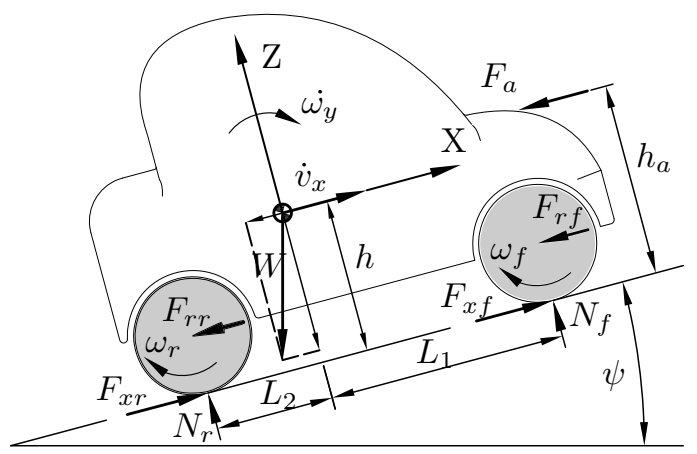

Figura 2: Diagrama de cuerpo libre para el modelo de dinámica longitudinal de un vehículo se desprecia la transferencia de carga originada por el movimiento de balanceo. A pesar de la simplicidad de este modelo, ha sido utilizado en multitud de trabajos donde lo primordial es la identificación de las fuerzas que afectan a un vehículo eléctrico en su desplazamiento rectilíneo, lo que permite realizar, entre otros estudios, predicciones de la potencia necesaria.

En la figura 2 muestra un esquema que representa un vehículo circulando sobre una carretera con cierta inclinación y las fuerzas a las que está sometido así como los movimientos que tienen lugar, siendo $\dot{h_{y}}$ la derivada del momento angular del vehículo en el eje $Y$ (eje de cabeceo) medido en el centro de gravedad del vehículo, donde se concentra su peso, representado por $W$. Las fuerzas de tracción y resistencia al avance están contenidas en el eje longitudinal del vehículo (eje $X$ ) siendo las fuerzas de resistencia a la rodadura de las ruedas delanteras y traseras $\left(\begin{array}{lll}F_{r f} & \text { y } & F_{r r}\end{array}\right)$, y las fuerzas de tracción en el eje delantero y trasero $\left(F_{x f}\right.$ y $\left.F_{x r}\right)$. Por su parte, las fuerzas de reacción normales delanteras y traseras se representan como $N_{f}$ y $N_{r}$, respectivamente. Finalmente, la fuerza aerodinámica se representa como una carga de resistencia al avance aplicada a una altura correspondiente a la del centro de gravedad del vehículo.

Una vez identificadas las fuerzas que actúan sobre un vehículo considerado como un sólido rígido, su dinámica longitudinal se puede expresar como:

$$
m_{v} \dot{v}_{x}=F_{x f}+F_{x r}-F_{r f}-F_{r r}-F_{a}-W \sin \psi
$$

El término $m_{v} \dot{v}_{x}$ se corresponde con la fuerza de aceleración y depende de la fuerza de propulsión $F_{x}$ (la suma de $F_{x f}$ y $F_{x r}$ ) menos las fuerzas de resistencia, compuestas por la fuerza de resistencia a la rodadura $F_{r}$ (igual a la suma de $F_{r f}$ y $F_{r r}$ ), la resistencia aerodinámica $F_{a}$ y la fuerza por pendiente $W \sin \psi$.

La fuerza de resistencia a la rodadura se produce por la histéresis en la deformación del neumático en su contacto con el asfalto, y depende de numerosos factores como son la presión de inflado, el tipo de neumático, las condiciones del pavimento o la velocidad. Esta fuerza se puede calcular mediante relaciones empíricas obtenidas a partir de ensayos experimentales [3]. Para ello, es necesario, en primer lugar, obtener el coeficiente de resistencia a la rodadura $f_{r}$, que para las condiciones operación en este trabajo, es decir, neumáticos para turismo y superficie asfaltada, se calcula como:

$$
f_{r}=f_{o}+f_{s}\left(\frac{v_{x}}{100}\right)^{2.5}
$$


siendo $f_{s}$ y $f_{s}$ dos coeficientes que dependen de la presión de inflado de los neumáticos. Si se considera un intervalo pequeño de variación de la presión de inflado, la ecuación 2 se puede aproximar a una función lineal con la velocidad:

$$
f_{r}=0.01\left(1+\frac{v_{x}}{160}\right)
$$

No obstante, para velocidades inferiores a 128 $\mathrm{km} / \mathrm{h}$, como es el caso en el presente trabajo, este coeficiente se puede considerar constante y con un valor de 0.015 [1]. Por tanto, la fuerza de resistencia a la rodadura se puede expresar como:

$$
F_{r}=0.015 W \cos \psi
$$

Por su parte, la fuerza aerodinámica de resistencia al avance se producen fundamentalmente por la presión que ejerce el aire al ser desplazado por la superficie frontal del vehículo y depende de numerosos factores como son la forma de la carrocería del vehículo, la inclinación del parabrisas o el tamaño de los neumáticos. Si bien no existen modelos teóricos precisos para calcular esta fuerza, se puede usar, de manera aproximada, la siguiente expresión:

$$
F_{a}=\frac{1}{2} \rho C_{x} A_{f}\left(v_{x}+v_{v}\right)^{2}
$$

siendo $A_{f}$ el área frontar del vehículo, definida a partir de la mayor sección transversal de éste, $\rho$ la densidad del aire, $v_{v}$ la velocidad del viento en oposición a la dirección de marcha y $C_{x}$ un coeficiente de resistencia aerodinámica que depende de la geometría del vehículo y para el presente trabajo se puede establecer un valor de 0.3 [3].

A partir de este modelo, se analizará la influencia de cada una de las fuerzas de oposición al movimiento, así como como la fuerza de aceleración lineal. Para ello, se llevará a cabo una simulación basada en datos experimentales que fueron registrados por los sensores del vehículo eCARM durante ensayos en diferentes maniobras. En esta simulación, el vehículo acelera desde un estado de reposo hasta que alcanza una determinada velocidad. Los datos de consumo eléctrico y velocidad fueron registrados y se muestran en la Figura 3a. Como se puede observar, para que el vehículo se ponga en marcha es necesaria una intensidad por encima de los $200 A$, elevándose el voltaje del motor por encima de $20 \mathrm{~V}$ hasta que se alcanza una velocidad de $6 \mathrm{~m} / \mathrm{s}$, a medida que la intensidad disminuye hasta que se alcanza el régimen estacionario.

En la Figura 3-b se muestran los resultados de

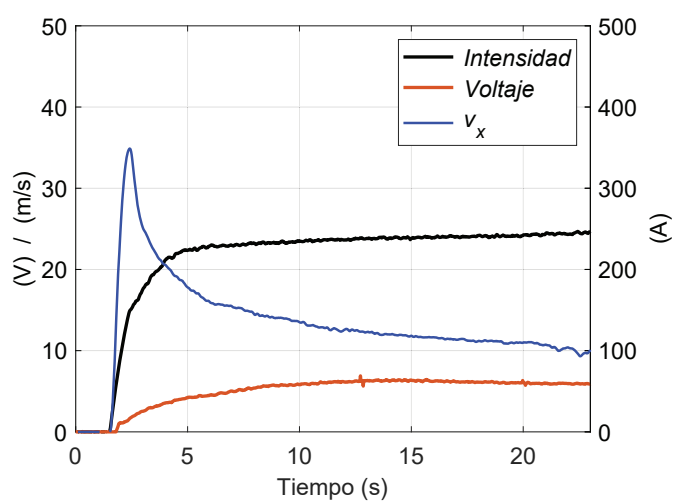

(a) Ensayo real

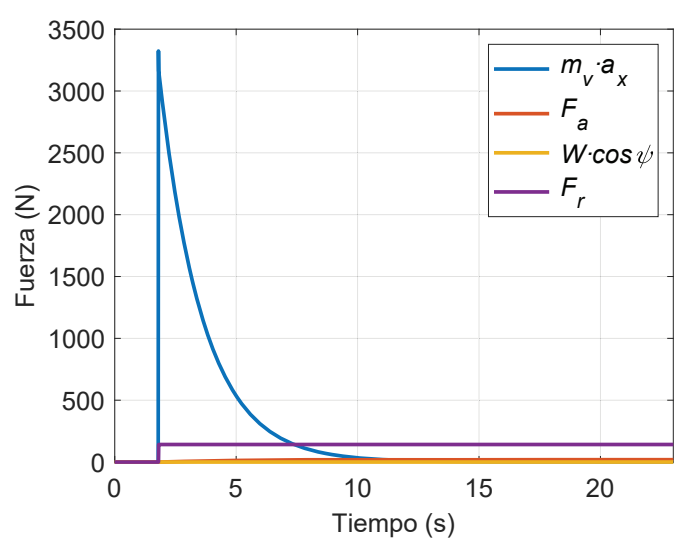

(b) Simulación

Figura 3: Ensayo de aceleración en línea recta. (a) Medida de la la intensidad eléctrica a través del motor eléctrico para un determinado voltaje y velocidad de marcha del vehículo. (b) Simulación de las fuerzas de acuerdo al modelo presentado en la sección 2.1

una simulación correspondiente a la misma maniobra desempeñada por un modelo de la dinámica longitudinal del vehículo. A partir de los datos obtenidos se desprende que la fuerza necesaria para poner el vehículo en movimiento es muy superior a las fuerzas de resistencia a la rodadura y aerodinámica, lo que se corresponde con el elevado consumo de corriente eléctrica registrado en la Figura 3-a en el arranque. Esta fuerza, identificada como $m_{v} a_{x}$ se corresponde con la fuerza de aceleración, y tiende a cero a medida que se alcanza la velocidad establecida. Una vez que esto ocurre, las fuerzas responsables del consumo eléctrico en régimen estacionario se corresponden, fundamentalmente, con la fuerza de resistencia a la rodadura y, en menor medida dada la baja velocidad en el ensayo, la fuerza de resistencia aerodinámica. 


\subsection{DINÁMICA ROTACIONAL: CÁLCULO DE LA INERCIA EQUIVALENTE}

En este apartado se presenta un método de cálculo de la inercia equivalente del vehículo para dimensionar el volante de inercia a incorporar en el banco de ensayos. Dicho cálculo se basará en la energía cinética del vehículo.

La energía cinética total de un vehículo en movimiento se compone por tanto de la energía cinética en desplazamiento traslacional y la energía cinética rotacional de los movimientos en rotación:

$$
E_{c}=\frac{1}{2} m_{v} v_{x}^{2}+\frac{1}{2} J \omega_{w}^{2}
$$

donde $\omega_{w}$ es la velocidad angular de los neumáticos y $J$ es la inercia rotacional de todos los componentes calculada en la rueda.

Esta energía se puede calcular como la energía cinética no rotacional de una masa equivalente $m_{e}$ que incorpora el incremento de inercia producido por los momentos angulares de los elementos en rotación del vehículo mediante la siguiente expresión:

$$
\frac{1}{2} m_{e} v^{2}=\frac{1}{2} m_{e} v_{x}^{2}+\frac{1}{2} J \omega_{w}^{2}
$$

Estableciendo la relación entre la velocidad angular de las ruedas y la velocidad lineal del vehículo según $v_{x}=\omega_{w} r_{w}$ y sustituyendo en la ecuación 7 se obtiene:

$$
m_{e}=m_{v}+J\left(\frac{1}{r_{w}}\right)^{2}
$$

A partir de la expresión anterior, se deduce que para calcular la masa equivalente, $m_{e}$, sería necesario obtener la inercia de todos los elementos mecánicos en el vehículo obteniendo el factor de inercia angular, $\delta$, mediante la siguiente expresión:

$$
\delta=1+\frac{I_{w}}{m_{v} r_{w}^{2}}+\frac{i_{0}^{2} i_{g}^{2} I_{p}}{m_{v} r_{w}^{2}}
$$

donde $I_{w}$ y $I_{p}$ representan la inercia angular de las ruedas y los elementos en rotación en el todo el sistema de propulsión y transmisión, siendo $i_{0}$ e $i_{g}$ sus respectivas relaciones de transmisión. Dado que difícilmente se va a disponer de de información precisa sobre $I_{w} \mathrm{y}$, especialmente, $I_{p}$, existe una aproximación empírica para determinar el factor de inercia de rotación mediante la siguiente ecuación:

$$
\delta=1+\delta_{1}+\delta_{2} i_{0}^{2} i_{g}^{2}
$$

en la que $\delta_{1}$ representa el segundo término del lado derecho de la ecuación 9 y $\delta_{2}$ representa el efecto de los elementos de rotación. Valores razonables de estos parámetros son 0.04 y 0.0025 respectivamente [3].

Una vez que se dispone del factor de inercia de rotación y conocida la relación de transmisión total, que en el caso del vehículo objeto de estudio es $i=9.75$, se puede calcular la masa equivalente como:

$$
m_{e}=m \delta
$$

Ahora es posible calcular la inercia rotacional equivalente, $J_{e}$, igualando la energía cinética de traslación con la energía cinética de rotación:

$$
\frac{1}{2} m_{e} v^{2}=\frac{1}{2} J_{e} \omega_{w}^{2}
$$

Esta sería la inercia equivalente rotacional calculada en las ruedas. No obstante, dado que se pretende calcular la inercia de la carga acoplada al motor eléctrico se debe tener en cuenta el número de vueltas que gira el eje de este por cada vuelta de las ruedas, esto es, $i=\omega_{m} / \omega_{w}$, por lo que sustituyendo esta expresión en la ecuación 12 y operando se obtiene la inercia en el eje del motor $J_{m}$ :

$$
J_{m}=\frac{m_{e} r^{2}}{i^{2}}
$$

\section{DISEÑO DEL BANCO EXPERIMENTAL}

Una vez que se dispone de la inercia que debe acoplarse al motor objeto de ensayo, se procede al diseño del banco experimental. Este consta de dos módulos: el primero sirve de soporte del motor, al que se conecta un freno de disco. Puesto que se pretende obtener una medida directa sobre las condiciones de trabajo del motor, este eje está acoplado a un sensor dinámico de par y a un encoder. El segundo módulo contiene el volante de inercia. Puesto que se ha intentado reproducir, con la mayor precisión posible, el régimen de funcionamiento del motor en el vehículo real, se instaló un sistema reductor de velocidad por cadena con la misma relación que aquella entre el motor y las ruedas $(i=9.75)$. Dado que es un factor elevado, el sistema reductor se compone de dos etapas. El último eje, que se corresponde con la salida de la segunda etapa, es el que contiene el 


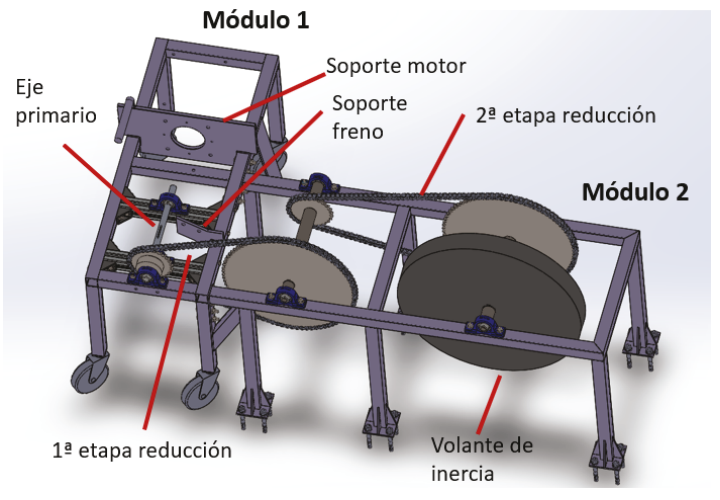

Figura 4: Diseño del banco de ensayos mediante herramientas CAD. Como se puede observar, el banco se compone de un primer módulo que contiene el eje primario que se conecta al motor, y un segundo módulo que incorpora el volante de inercia y el sistema transmisión.

volante de inercia cuyo valor es igual a la inercia equivalente calculada en la sección 2.2 menos la inercia de los elementos que componen el sistema de transmisión.

Cabe destacar que en el cálculo de la inercia equivalente planteada en la sección 2 se consideró que el volante de inercia se acoplaba directamente al eje de salida del motor, tal y como refleja la ecuación 13. No obstante, de acuerdo al diseño final del banco, el volante de inercia no se conecta directamente al eje primario, sino que existe una relación de transmisión entre éste y el eje que contiene al volante de inercia. Teniendo en cuenta esto, se puede calcular la inercia del volante $J_{v}$ mediante la siguiente expresión:

$$
J_{v}=\frac{m_{e} r^{2}}{i}
$$

considerando que el radio de la rueda es $r_{w}=$ $0.2805 \mathrm{~m}$ y la masa del vehículo es $m_{v}=740 \mathrm{~kg}$, se obtiene valor de inercia del volante situado en el eje secundario de $J_{v}=7.63 \mathrm{kgm}^{2}$.

\section{ENSAYOS Y RESULTADOS PRELIMINARES}

Una vez validado el diseño presentado en la sección 3 se procedió a su fabricación y montaje. En la Figura 5 se muestra el resultado final. Como se puede observar, el banco cuenta con sensores de medida eléctrica, como son los amperímetros y voltímetros, con los que se obtienen valores de consumo, así como un sensor de para medir el par entregado por el motor y un encoder que mide la velocidad de giro del eje primario.

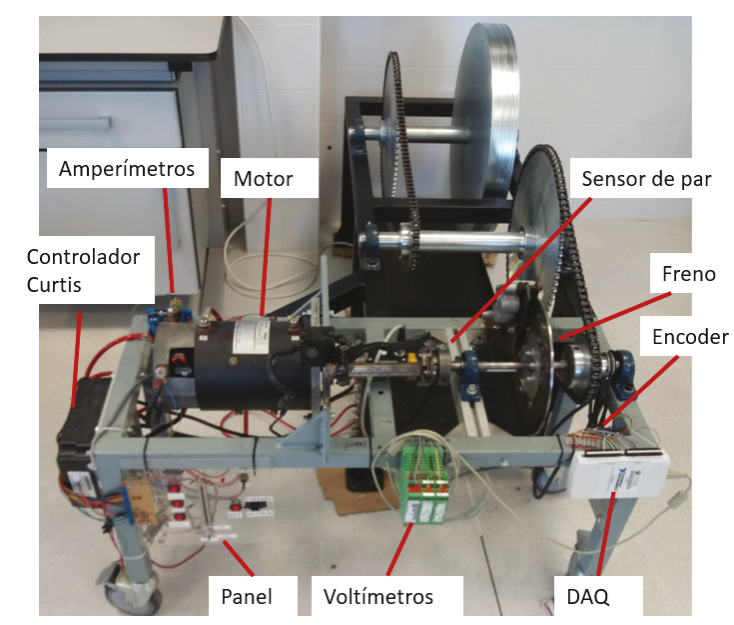

Figura 5: Imagen del banco experimental construido e identificación de los principales sensores y actuadores.

Las señales de estos sensores son registradas por medio de una tarjeta de adquisición de datos (DAQ) modelo NI-USB 6210 del fabricante $\mathrm{Na}$ -
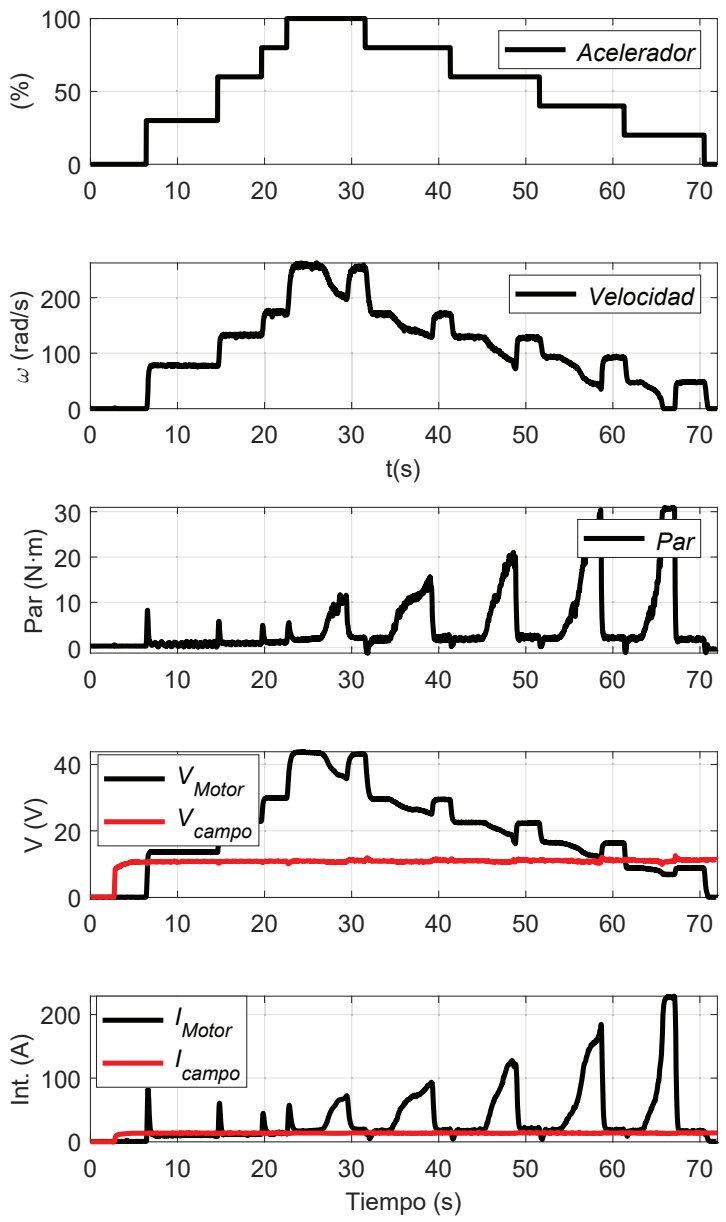

Figura 6: Ensayo bajo diferentes consignas de velocidad y actuación arbitraria del sistema de frenado. 

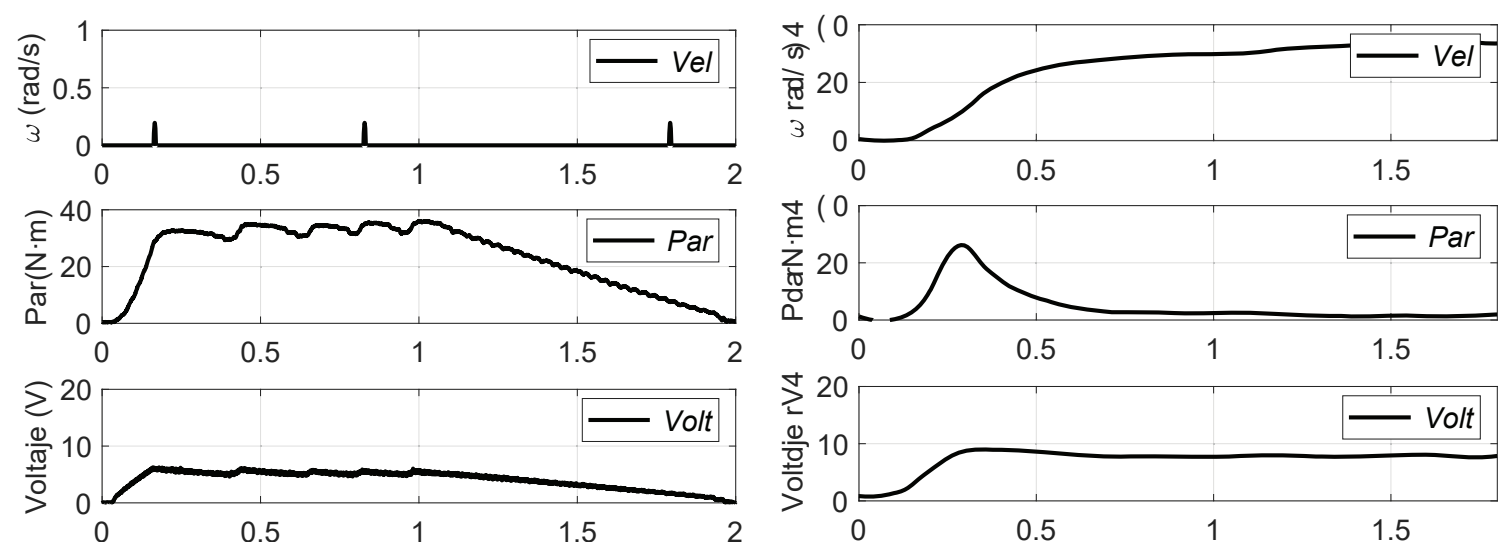

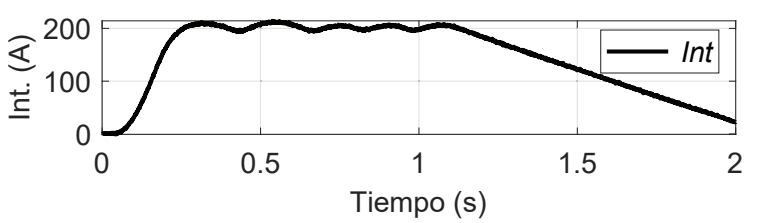

(a) Eje bloqueado

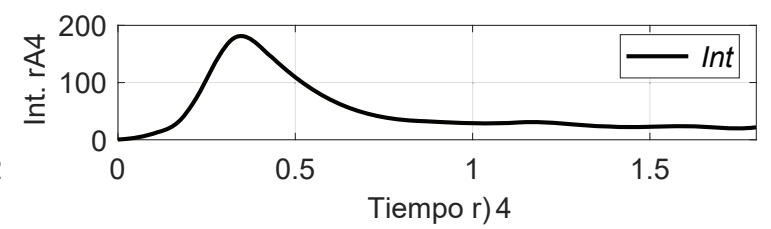

(b) Con volante de inercia

Figura 7: Ensayo de aceleración en línea recta. (a) Medida de la la intensidad eléctrica a través del motor eléctrico para un determinado voltaje y velocidad de marcha del vehículo. (b) Simulación de las fuerzas de acuerdo al modelo presentado en la sección 2.1

tional Instruments que se comunica con un PC haciendo uso de la Data Acquisition Toolbox de $M A T L A B^{\circledR}$. Esta toolbox ofrece una serie de funciones para la adquisición y envío de datos a partir de la definición del tiempo de muestreo, la frecuencia de muestreo, el canal de lectura y escritura y el tipo de dato esperado en el caso de la lectura para su tratamiento previo. Para poder hacer uso de estas funciones se ha de crear una sesión de adquisición de datos, donde previamente a la adquisición se han de definir todos los canales que se van a usar en esa sesión y el comportamiento de los mismos. Posteriormente se registran o publican los datos por un intervalo de tiempo, obteniéndose una matriz numérica de $m$ muestras por $n$ señales. Una de las variables de salida se corresponde con el accionamiento del acelerador del motor, a través de una señal analógica entre 0 y 5 $V$. Esta señal de referencia, equivalente a la que genera el pedal del acelerador cuando el motor está montado en el vehículo, constituye la entrada en el controlador del amplificador Curtis, específico para este tipo de motores, cuyo comportamiento hay que tener en cuenta a la hora de diseñar sistemas de control en cascada. De esta forma se pueden programar ensayos garantizando la repetitividad de los mismos. Si bien está prevista la instalación de un banco de baterías que alimente a dicho controlador y permita realizar estudios sobre el modelado de las mismas y su integración en una micro-red, en esta primera fase de desarrollo se utilizaron fuentes de alimentación conmutadas conectadas a una toma de corriente trifáisca. Por último, en la Figura 5 también se puede observar que el banco cuenta con un panel de mando manual por medio del cual se configuran los modos de funcionamiento del motor y se pueden introducir consignas mediante un potenciómetro.

Con objeto de verificar el correcto funcionamiento del banco de ensayos, se llevaron a cabo una serie de experimentos cuyos resultados se mostrarán a continuación. El primero de los ensayos consiste en una secuencia programada de consignas con el motor en vacío (desacoplado del segundo módulo y solo con la inercia propia del rotor, el eje junto con los acoplamientos al sensor de par y el disco de freno) y con actuaciones aleatorias sobre el sistema de frenado. A partir de los datos obtenidos, representados en la Figura 6 se pueden extraer ciertas conclusiones que ponen de manifiesto el correcto funcionamiento del mismo y que concuerdan con las que se obtienen en el resto de ensayos realizados. La primera es que mientras que el voltaje y la corriente en el inducido son constantes, el voltaje del motor está relacionado con su velocidad angular que mediante por la acción del controlador se corresponde con las consignas de entrada al mismo tiempo que la corriente del motor se relaciona con el par que debe vencer el motor para compensar las fuerzas de frenado y mantener la velocidad demandada, y, en menor medida debido a la baja inercia en este ensayo, a las aceleraciones requeridas. Cabe mencionar que la velocidad de giro del motor que se llega a alcanzar en este ensayo sería tal que el vehículo circularía a una velocidad alrededor de 
$20 \mathrm{~km} / \mathrm{h}$.

El segundo ensayo realizado consiste en la medida del consumo eléctrico y el par entregado manteniendo el sistema de frenado activado de manera que el motor esté bloqueado. Los resultados obtenidos se muestran en la Figura 7 (a). Como se puede apreciar, los valores de par entregado por el motor llegan a alcanzar los $35 \mathrm{Nm}$, y los de corriente $200 A$. También se puede apreciar que el voltaje apenas excede los $5 \mathrm{~V}$. Por último, comentar que los pulsos que se aprecian en la velocidad angular se deben a pequeños deslizamientos del disco de freno debidos al alto par al que está sometido.

El tercer y último ensayo consiste en una aceleración del motor partiendo de un estado de reposo conectado al segundo módulo del banco y por tanto con una carga equivalente a la del vehículo real. Los resultados obtenidos se muestran en la Figura 7 (b). Si bien la velocidad de giro del motor en este ensayo es bastante baja, no alcanzando siquiera los $40 \mathrm{rad} / \mathrm{s}$, el para necesario para poner el volante de inercia en movimiento llega a exceder los $20 \mathrm{Nm}$ originando una intensidad de corriente cercana a los $200 \mathrm{~A}$. El hecho de que la velocidad sea tan baja también se traduce en un reducido voltaje en el motor.

\section{CONCLUSIONES}

En este trabajo se ha presentado el diseño de un banco de ensayos de motores de vehículo eléctrico para la realización de experimentos sobre un motor perteneciente a un vehículo eléctrico que forma parte de una microrred eléctrica con presencia de energía fotovoltaica y almacenamiento. El banco de ensayos propuesto permite realizar dichos experimentos en las mismas condiciones en las que opera cuando está montado en el vehículo real, por lo que los resultados obtenidos en términos de rendimiento del motor y consumo eléctrico son válidos para la implementación de estrategias de control. En este trabajo se han realizado tres ensayos diferentes. En el primero, el eje primario permaneció desconectado del volante de inercia y se actuó sobre el sistema de frenado de forma arbitraria. En el segundo ensayo se bloqueó el eje primario mediante la actuación sobre el sistema de frenado y se aplicó una consigna sobre el acelerador. El tercer ensayo consistió en la puesta en marcha del motor desde un estado de reposo estando conectado al volante de inercia. En todos los resultados se obtienen unos valores de par, velocidad, voltaje e intensidad eléctrica que concuerdan con los resultados obtenidos en ensayos sobre el vehículo real y sobre los modelos en simulación. Como trabajos futuros se plantea la integración del banco de ensayos junto con sus baterías en una microrred, por un lado, y por el otro la elaboración de modelos precisos tanto del motor como de las baterías, a partir de ensayos que permitan su calibración y la implementación de estrategias de control en el vehículo real.

\section{Agradecimientos}

Este trabajo fue financiado en parte por el Proyecto de Plan Nacional de I + D + i DPI201785007-R del Ministerio de Economía, Industria y Competitividad y fondos FEDER.

\section{English summary}

\section{TEST BENCH FOR ELECTRIC VEHICLES' MOTORS}

\begin{abstract}
In this paper, a novel test bench for electric vehicles' motor which allows to reproduce the working conditions of a vehicle in terms of torque and angular speed considering its equivalent inertia is designed. This test bench allows, on the one hand, to develop models of both the motor and the batteries, as well as to design and test controllers and, on the other, the integration of an electric vehicle into an experimental electric microgrid in order to test energy management strategies without needing the real vehicle. According to the results obtained, it can be concluded that the proposed design meets the requirements.
\end{abstract}

Keywords: Test bench, electric vehicle, motor control.

\section{Referencias}

[1] F. Aparicio Izquierdo, C. Vera Alvarez, and V. Diaz Lopez. Teoria de los vehiculos automoviles. Escuela Tecnica Superior de Ingenieros Industriales. Universidad Politecnica de Madrid, 2001.

[2] C. Bordons, F. García-Torres, and L. Valverde. Gestión Óptima de la Energía en Microrredes con Generación Renovable. RIAI - Revista Iberoamericana de Automatica e Informatica Industrial, 12(2):117-132, 2015. 
[3] M. Ehsani. Modern electric, hybrid electric, and fuel cell vehicles : fundamentals, theory, and design. CRC Press, 2005.

[4] P. Fajri, R. Ahmadi, and M. Ferdowsi. Equivalent vehicle rotational inertia used for electric vehicle test bench dynamic studies. In IECON 2012 - 38th Annual Conference on IEEE Industrial Electronics Society, pages 4115-4120. IEEE, oct 2012.

[5] A. Fotouhi, S. Longo, K. Propp, L. Samaranayake, and D. Auger. A hardware-in-theloop test rig for development of electric vehicle battery identification and state estimation algorithms. International Journal of Powertrains, 7(1/2/3):227, 2018.

[6] T. J. Hassell, A. M. Oliveira, and W. W. Weaver. Design, construction, and testing of an electric machine test-bed for use in laboratory and research education. Proceedings - Frontiers in Education Conference, FIE, pages 470-476, 2013.

[7] S. Khan, K. Mehmood, Z. Haider, S. Bukhari, S.-J. Lee, M. Rafique, and C.-H. Kim. Energy Management Scheme for an EV Smart Charger V2G/G2V Application with an EV Power Allocation Technique and Voltage Regulation. Applied Sciences, 8(4):648, 2018.

[8] P. Renato, J. E. Normey-rico, C. B. Alba, and L. Valverde. Gestión energética de una Micro Red acoplada a un sistema V2G. In XXXVI Jornadas de Automática, pages 2-4, Bilbao, 2015.

[9] L. Simoni, M. Beschi, D. Colombo, A. Visioli, and R. Adamini. A Hardware-In-the-Loop setup for rapid control prototyping of mechatronic systems. IEEE International Conference on Emerging Technologies and Factory Automation, ETFA, 2015-Octob, 2015.

[10] Y. Sun, Y. Wang, R. Zhu, R. Geng, J. Zhang, D. Fan, H. Wang, J. Sun, and L. Wang. Development of test bed of hybrid electric vehicle based on chassis dynamometer. IOP Conference Series: Materials Science and Engineering, 452(4), 2018.

[11] J. Torres-Moreno, A. Gimenez-Fernandez, M. Perez-Garcia, and F. Rodriguez. Energy Management Strategy for Micro-Grids with PV-Battery Systems and Electric Vehicles. Energies, 11(3):522, feb 2018.
(C) 2019 by the authors. Submitted for possible open access publication under the terms and conditions of the Creative Commons Attribution CC BY-NC-SA 4.0 license (https://creativecommons.org/licenses/by-ncsa/4.0/deed.es). 